\title{
Type 4 hypersensitivity development in a case due to mifamurtide
}

\author{
Meral Şimşek ${ }^{1 \oplus}$, Erman Ataş ${ }^{2 \oplus}$, Emin Ümit Bağrıaçık $^{3 \odot}$, Armağan Günal ${ }^{4 \oplus}$, \\ Bülent Ünay ${ }^{5 \odot}$
}

Divisions of ${ }^{2}$ Pediatric Hematology and Oncology and ${ }^{5}$ Pediatric Neurology, ${ }^{1}$ Department of Pediatrics, ${ }^{4}$ Department of Pathology, University of Health Sciences, Gülhane Faculty of Medicine, Ankara; ${ }^{3}$ Department of Immunology, Gazi University Faculty of Medicine, Ankara, Turkey.

\begin{abstract}
Background. This report aims to discuss the mechanism of pleural and pericardial effusion related to mifamurtide which is an immunological agent used as adjuvant chemotherapy in osteosarcoma.

Case. Mifamurtide $\left(2 \mathrm{mg} / \mathrm{m}^{2}\right)$ and European and American Osteosarcoma Studies (EURAMOS) protocol were used together intravenously after complete surgical resection. No side effects occurred except for fever after the first dose. However, pleural, pericardial effusion, and splenic nodule formation began 11 months after discontinuation of mifamurtide treatment. Pleural biopsy revealed a type 4 hypersensitivity reaction. We treated the patient with 1,5 mg per day colchicine. Pericardial effusion attacks and nodules in the spleen disappeared. The patient had a mild pleural effusion attack which has not yet repeated.

Conclusion. Mifamurtide, which activates macrophages, can also activate immunity with a stand by effect and cause a hypersensitivity reaction.
\end{abstract}

Key words: child, osteosarcoma, mifamurtide, pleural effusion, pericardial effusion, hypersensitivity.

Osteosarcoma (OS) is the most common bone tumor in childhood. Considerable progress in survival has been obtained recently. Advances in chemotherapy, surgery, and immunotherapy have shown their influence in the treatment of OS. Mifamurtide is an immunological agent used in the treatment of OS. ${ }^{1}$ It influences treatment at the level of micrometastasis. However, there are some side effects as well as positive contributions to treatment. Most of them are chills, fever, headache. Mifamurtide can affect all systems in the body. However, some of these side effects maybe life threatening. ${ }^{2}$ Thus, we tried to reveal the pharmacodynamical mechanism of side effects in a patient who developed pleural and pericardial effusion attacks after mifamurtide.

凶 Meral Şimşek

meralulgersimsek@gmail.com

Received 7th January 2020, revised 24th February 2020, accepted 17th March 2020.

\section{Case Report}

A 10-year-old male patient was admitted with left knee pain. Radiological examination of the left knee revealed a $9 \times 5.5 \times 4 \mathrm{~cm}$ mass. A biopsy was performed, and the pathology was consistent with high-grade OS. After a 10week preoperative European and American Osteosarcoma Studies (EURAMOS) protocol, limb-sparing surgery was performed. Mifamurtide was added to the postoperative EURAMOS treatment. Treatment was discontinued in complete remission. Approximately 11 months after mifamurtide treatment, he was brought in with complaints of chest pain, back pain, and dyspnea. Thorax ultrasonography revealed a $5.5 \mathrm{~cm}$ pleural effusion that collapsed the lung in the left thorax. Echocardiography showed moderate pericardial effusion, which was approximately $15 \mathrm{~mm}$ in the anterior and posterior walls. It also included fibrin particles. Purified protein 
derivative (PPD) was anergic. A chest tube was inserted. Vancomycin and ceftriaxone were started for complicated pneumonia. During the follow-up, vancomycin and meropenem treatments were started due to the fact that fever and acute phase elevation persisted. In the pleural fluid biochemical examination results were measured as glucose: $135 \mathrm{mg} / \mathrm{dl}$, concurrent glycaemia: $114 \mathrm{mg} / \mathrm{dl}$, protein: $5.7 \mathrm{mg} / \mathrm{dl}$, serum protein: 4,82 mg/dl, LDH: $1674 \mathrm{U} / \mathrm{L}$, serum LDH: 303U/L. Cytology revealed abundant neutrophils, leukocytes, and histiocytes. Pericardiocentesis was performed. Pericardial cytology also showed diffuse lymphocytes, plasma cells, and macrophages. In a pericardial biopsy, chronic inflammation and fibrosis tissue were detected. Cultures did not grow, and no Adenosine Deaminase Activity (ADA) activity was detected. Mycobacteria culture was negative. Pericardial effusion regressed to $11 \mathrm{~mm}$ in the second week of treatment. Abdominal ultrasound showed a slightly heterogeneous, hyperechoic, nonspecific area. It was barely distinguishable from the normal spleen parenchyma. On computer tomography (CT), a solid lesion with a diameter of $3 \mathrm{~cm}$ was confirmed, and fluorodeoxyglucose -positron emission tomography with computer tomography (FDG PET / CT) and bone scintigraphy showed no significant increase in metabolic activity. A biopsy was not performed. After this event, the patient was brought back with the same complaints five months later. Pleural effusion was seen on chest X-ray, and thorax ultrasonography was compatible with $25 \mathrm{~mm}$ pleural effusion. Echocardiography showed a size of $7 \mathrm{~mm}$. Bilateral pericardial effusion revealed pericarditis. A chest tube was inserted. The pericardial window was opened. Vancomycin, ceftriaxone, and antiinflammatory ibuprofen treatments were initiated. Clarithromycin was added when fever and acute phase elevation persisted. Exudate or transudate was not differentiated from the pleural fluid sample. Pleural biochemical examination revealed $\mathrm{pH}: 7.8, \mathrm{LDH}: 200 \mathrm{U} / \mathrm{L}$, serum LDH: $155 \mathrm{U} / \mathrm{L}$, glucose: $160 \mathrm{mg} / \mathrm{dl}$, serum glucose: $95 \mathrm{mg} / \mathrm{dl}$, protein: $5.3 \mathrm{mg} / \mathrm{dl}$, serum protein: $6.75 \mathrm{mg} / \mathrm{dl}$. The pleural fluid culture was negative. Mycobacteria culture was negative, and no ADA activity was detected. The repeated PPD test was anergic. Cytological examination revealed no tumor cells. Pleural fluid smear showed abundant neutrophils, leukocytes, and histiocytes. ANA and Anti-ds DNA obtained for the etiology were negative. Urinary calcium, serum ACE level, and eye examination for sarcoidosis were normal. Genetically, FMF gene analysis was reported negative. The nodule in the spleen persisted with the same characteristics. There was no malignant involvement in repeated PET/CT. In a pericardial biopsy, chronic inflammation and fibrotic tissue were detected. Pleural biopsy revealed diffuse fibrotic tissue with focal mesothelial cell hyperplasia and congestion and no malignant involvement. The pleural biopsy specimen was stained with CD3 staining, and intense $\mathrm{T}$ cell foci were detected. CD19 stain revealed approximately 3-4 focal B lymphocyte foci in each area (Fig. 1). The patient was treated with colchicine $3 \times 0.5 \mathrm{mg}$ and had a new pleural effusion attack five months after the treatment was started. However, he did not have a concomitant pericardial effusion and regressed with outpatient antibiotic therapy. Besides, abdominal ultrasonography performed during this period showed that the nodule in the spleen disappeared. It was observed that pleural and pericardial effusion attacks did not reoccur. The patient is still in complete remission.

Informed consent was received from the family for writing this case.

\section{Discussion}

Successful treatment of non-metastatic, metastatic, and recurrent OS has been obtained with multi-agent chemotherapy, and local control procedures such as amputation, limb salvage surgery with negative margins, surgical removal of lung/bone metastases. ${ }^{3}$ Because of the low survival rate for those with metastatic disease, treatment opportunities before metastasis, that activate the immune 


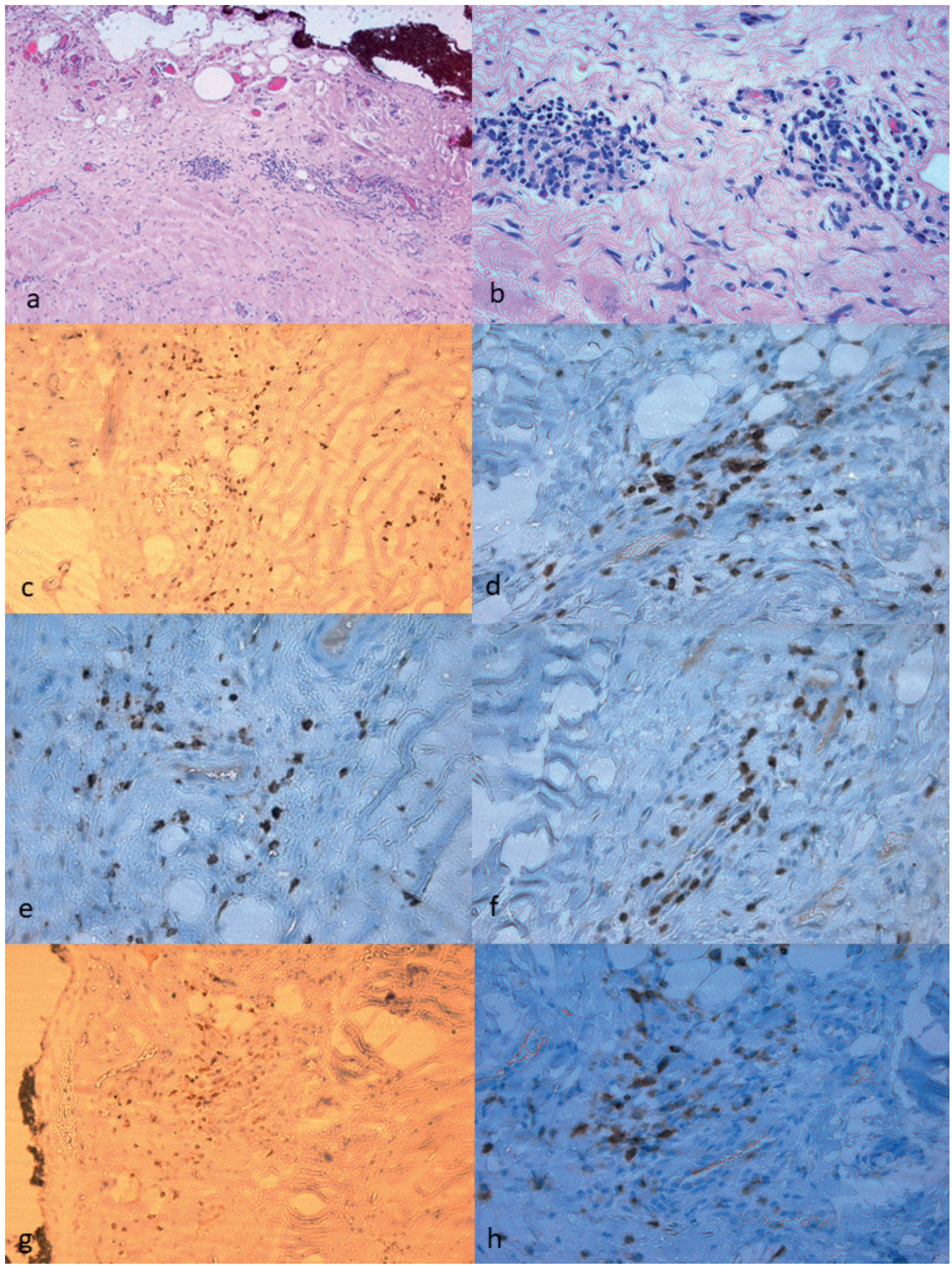

Fig. 1. (a) Intensive lymphocyte infiltration with HEx100 in pleural tissue biopsy specimen, (b) Focus of lymphocyte inflltration with HEx400, (c) T lymphocyte infiltration foci with IHC-CD3x100 in pleural tissue biopsy sample, (d, e, f) Intense infiltration of T lymphocytes with IHC-CD3x400, (g) B lymphocyte infiltration foci with IHC-CD19x100 in pleural tissue biopsy specimen, (h) B lymphocyte infiltration with IHC-CD19. 
system, contributing to better outcomes, have been investigated. Infections associated with limb surgery have a positive effect on survival in humans with OS. ${ }^{3}$ Higher absolute lymphocyte count recovery after chemotherapy also appears to be associated with improved survival. ${ }^{3}$ In the phase 3 of the CCG/POG study where mifamurtide was begun after definitive surgery whether it was used or not, the addition of Liposomal-Muramyl TriPeptidePhosphatidylEthanolamine (L-MTP-PE) made progress in event free survival (EFS) $(\mathrm{p}=0.08)$, and had a significant improvement in OS $(78 \%$ vs. $70 \% ; p=0.03) .{ }^{4}$ EURAMOS used interferon as immunotherapy in the good responders. The addition of interferon did not produce improvement of EFS. ${ }^{5}$ Thus, we decided to use the EURAMOS protocol and add mifamurtide postoperatively in non-metastatic patients. To sum up, the addition of mifamurtide to treatment with chemotherapy may have some beneficial effects on metastatic or nonmetastatic patients via eradication of residual micrometastases that are not eliminated by chemotherapy alone. Activated monocytes remain tumoricidal for up to 96 hours following mifamurtide infusion. ${ }^{6}$ Mifamurtide does not accumulate during repeated administration.

When liposomes are applied systematically, they are accumulated by the phagocytic cells of the mononuclear phagocyte system, which is primarily in the liver, spleen, nasopharynx, thyroid, and lungs. ${ }^{7}$ Muramyl dipeptide (MDP), the smallest component of immune potentiating activity from the cell wall of the Bacille Calmette-Guerin, stimulates innate immunity. The liposomal MTP-PE (L-MTP-PE) has similar immunostimulatory effects as natural MDP, with the additional advantage of a longer half-life in plasma and lower toxicity. The encapsulation of MTP-PE into liposomes has been shown invitro to enhance the activation of macrophages by 100 -fold compared with free MDP. ${ }^{8}$ Possible mechanism of mifamurtide can be muramyl dipeptide ligand to NOD2 receptors. In addition, lipoprotein on MTP-PE ligand to Tolllike receptors which are recognition receptors at the monocytes and macrophages is the other mechanism. Thus it forms a vesicle from the cell membrane and enters the cell. It activates the transcription factor $\mathrm{NF \kappa B}$ via the intracellular domain MyD88 and activates protein synthesis by binding to the promoter region of DNA. ${ }^{1}$ Normally, active monocytes turn to active macrophages, also called histiocytes in tissue. However, activated monocyte with mifamurtide in blood act like it's in tissue and works like a macrophage. In this stage, the release of cytokines, such as IL-1, IL-6, IL-8, IL12, and TNF-alfa, IFN-gamma, called 'cytokine flu' occurs. Meanwhile, muramyl dipeptide epitopes bind to major histocompatibility complex (MHC) molecules and serve to the cell membrane. Antigen-presenting cell serves antigenic epitope to $\mathrm{T}$ and $\mathrm{B}$ lymphocytes in closest secondary lymph node follicles, as in the spleen follicles. Once T lymphocytes recognize MHC, they start to make permanent contact by adhesion molecules. For the first signal, ICAM1 \& LFA-1 adhesion molecules, a complex of "CD4 or CD8+T cell receptor (TCR)+CD4" and compatible MHC molecule reaction takes place. The second signal between CD28, and CD80/86 and the third signal provides differentiation and triggers the generation of Th1, Th2, Th17, and $\mathrm{T}$ regulatory. The active macrophages make a 'stand by' effect for overall immunoreactions. Within 96 hours, they react maximum, and then the impact of $\mathrm{T}$ cells reduces. Also, some of them last for years as T memory. MTP-PE shows adjuvant 'stand by' effect for $\mathrm{T}$ cells growth.

Activated macrophages move to that area via the chemokines, which are secreted by the antigen-presenting cell on tumoral tissue. Immune cells recognize the apoptotic signals like Phosphatidylserine (PtdSer), which translocated to the leaflet of tumor cells membrane with eat-me messages. ${ }^{9}$ This is a macrophage-mediated tumoricidal effect. Similar to our study, some studies have reported that human macrophages after invitro activation with mifamurtide specifically recognize tumor cells and are not cytotoxic to healthy cells. ${ }^{6,8}$ Similar to our research, one study reported 
that anti-tumor activity is linked to both direct and indirect effects of macrophage activation, including contact-mediated tumor cytotoxicity and release of tumoricidal factor. ${ }^{10}$

The literature reports that mifamurtidine has some side effects, mostly potential infusion-related side effects. ${ }^{2}$ Common side effects include fever, chills, headache nausea, tachycardia, vomiting, hypotension, hypothermia, dyspnea, constipation, and pain. ${ }^{11}$ The severe side effects such as acute respiratory distress, hypoxia, malignant pleural effusion related to renal dysfunction, hepatic dysfunction, pyrexia, pericardial effusion (resolved in 1 week), respiratory distress, arrhythmia, peripheral sensory and centro- cerebellar neural involvement, skin disorders, ear disorders hearing-objective, febrile neutropenia, thrombocytopenia lead to discontinuation of drug. ${ }^{8}$ However, there is limited data concerning the toxicity mechanism of mifamurtide in children with OS. In a phase 2 study, 12 patients aged 9-21 years with histologically proven OS with primary tumor resected that developed resectable metastases during or after adjuvant chemotherapy or those who initially presented with metastases that persisted despite chemotherapy were evaluated for side effects. Pleuritis and pericarditis were observed in 2 patients. ${ }^{11}$ This status was similar to our case. In a study, 678 patients developed hypersensitivity reactions along with pleural and pericardial effusions, seizures, and muscle spasms. ${ }^{12}$ Anderson et al. $^{3}$ also reported the side effects. They were generally Grade 1 or Grade 2 in $49 \%$ of cases. However, $2 \%$ of the patients had rare side effects potentially related to mifamurtide, such as pericardial and pleural effusions. Thus, we aimed to reveal the mechanism of pharmacodynamics and rare side effects of mifamurtide.

In our case, the patient had relapsing pericardial and pleural effusions which we believe may have occurred due to mifamurtide, since other etiological factors were negative. In the literature hypersensitivity reactions such as pleural and pericardial effusions, seizures, and muscle spasms, and severe hearing loss are mentioned. ${ }^{12}$ Because of the lack of any survival benefit and the risk of serious adverse effects, the addition of mifamurtide to chemotherapy regimens is not advised. Similar to reports our patients had a severe side effect. Our patient's pathology of the pleural biopsy revealed so much focus on $\mathrm{T}$ cells and 3-4 focus in each area of B cells with diffuse fibrosis. It was compatible with type 4 hypersensitivity reactions. No macrophages were seen morphologically. Macrophage formation can be deformed. This may occur is the biopsy material is obtained a long time after the beginning of the inflammation. The absence of macrophages can also be attributed to the change of morphology of macrophages in the advanced stage of inflammation. Since the investigations for the etiology of this status were all normal. This clinical picture was thought to be due to the rare side effects of mifamurtide, an adjuvant immunological agent given to the patient. A type 4 hypersensitivity reaction was considered mainly. This hypersensitivity reaction may be caused by the inability to stop mifamurtide-induced immunoreactivity initiated by the 'stand by' effect. Primarily formed T memory and B memory cells may be effective in the formation of late-period type 4 hypersensitivity reactions. This autoimmune reaction may be due to the dose of the drug, route of administration, or the patient's immunity. Thus, MTP-PE can trigger hypersensitivity in some patients. The nodular lesion on spleen parenchyma may have also occured due to mifamurtide, but a biopsy was not performed for the splenic nodule. This exaggerated immune response was reduced by colchicine. Pericardial effusion attacks and nodules in the spleen also disappeared. However, like in our case the antiinflammatory effect of drugs, such as ibuprofen may not prevent the progression of the attack, because non steroid anti-inflammatory drugs do not inhibit leucocytes, which are responsible for initiating inflammation. Besides, the effect on COX1>COX2 can be accountable for this. ${ }^{13}$ Colchicine inhibits neutrophil activation and migration to the inflammation center. It also prevents microtubule, inflammasome, 
and microtubule related chemotaxis, and phagocytosis. ${ }^{14}$ TNF- $\alpha$ receptors on the surface of macrophages and endothelial cells are decreased by colchicines. ${ }^{15}$ Although the patient had a mild pleural effusion attack, it has not yet repeated. This showed us that colchicine has a positive effect on inflammation.

In conclusion, mifamurtide is generally welltolerated, with some side effects reported as either mild or moderate. However, it can cause hypersensitivity reactions. Future studies should focus more on the mechanism of the hypersensitivity reactions. Also, mifamurtide may be combined with PD-L1-Fc, which are regulation molecules for regulation of $\mathrm{T}$ cells. In addition, encapsulation with an inhibitory cytokine which TGF- $\beta 1$ may increase transformation to $\mathrm{T}$ regulatory cells directly in order to reduce autoimmune reactions.

\section{REFERENCES}

1. Kansara M, Teng MW, Smyth MJ, Thomas DM. Translational biology of osteosarcoma. Nat Rev Cancer 2014; 14: 722-735.

2. Jimmy R, Stern C, Lisy K, White S. Effectiveness of mifamurtide in addition to standard chemotherapy for high-grade osteosarcoma: a systematic review. JBI Database System Rev Implement Rep 2017; 15: 2113-2152.

3. Anderson PM, Meyers P, Kleinerman E, et al. Mifamurtide in metastatic and recurrent osteosarcoma: a patient access study with pharmacokinetic, pharmacodynamic, and safety assessments. Pediatr Blood Cancer 2014; 61: 238-244.

4. Meyers PA, Schwartz CL, Krailo MD, et al; Children's Oncology Group. Osteosarcoma: the addition of muramyl tripeptide to chemotherapy improves overall survival--a report from the Children's Oncology Group. J Clin Oncol 2008; 26: 633-638.
5. Bielack SS, Smeland S, Whelan JS, et al; EURAMOS-1 investigators. Methotrexate, doxorubicin, and cisplatin (MAP) plus maintenance pegylated interferon alfa- $2 b$ versus MAP alone in patients with resectable high-grade osteosarcoma and good histologic response to preoperative MAP: first results of the EURAMOS-1 good response randomized controlled trial. J Clin Oncol 2015; 33: 2279-2287.

6. Frampton JE. Mifamurtide: a review of its use in the treatment of osteosarcoma. Paediatr Drugs 2010; 12: 141-153.

7. Murray JL, Kleinerman ES, Cunningham JE, et al. Phase I trial of liposomal muramyl tripeptide phosphatidylethanolamine in cancer patients. J Clin Oncol 1989; 7: 1915-1925.

8. Meyers PA. Muramyl tripeptide (mifamurtide) for the treatment of osteosarcoma. Expert Rev Anticancer Ther 2009; 9: 1035-1049.

9. Lemke G. How macrophages deal with death. Nat Rev Immunol 2019; 19: 539-549.

10. Thundimadathil J. Cancer treatment using peptides: current therapies and future prospects. J Amino Acids 2012; 2012: 967347.

11. Ando K, Mori K, Corradini N, Redini F, Heymann D. Mifamurtide for the treatment of non-metastatic osteosarcoma. Expert Opin Pharmacother 2011; 12: 285-292.

12. Mifamurtide: osteosarcoma: ineffective and harmful. Prescrire Int 2011; 20: 89.

13. Schwier N, Tran N. Non-steroidal anti-inflammatory drugs and aspirin therapy for the treatment of acute and recurrent idiopathic pericarditis. Pharmaceuticals (Basel) 2016; 9: E17.

14. Dalbeth N, Lauterio TJ, Wolfe HR. Mechanism of action of colchicine in the treatment of gout. Clin Ther 2014; 36: 1465-1479.

15. Ding AH, Porteu F, Sanchez E, Nathan CF. Downregulation of tumor necrosis factor receptors on macrophages and endothelial cells by microtubule depolymerizing agents. J Exp Med 1990; 171: 715727. 